\title{
DIABETES GESTACIONAL
}

\author{
Marcela Otálora MD*, Gina Milena Ortiz MD**
}

\section{Resumen}

La diabetes gestacional ocurre entre dos y quince por ciento de los embarazos. Los factores de riesgo incluyen obesidad, intolerancia a la glucosa, historia familiar de diabetes mellitus y antecedente de óbito fetal. El diagnóstico se sustenta en la curva de tolerancia a la glucosa con dos valores anormales. El manejo médico se basa en dieta hipocalórica y ejercicio La terapia farmacológica con insulina ha sido tradicional. Los análogos de insulina de acción rápida se aprobaron en 1996. Se analizan el seguimiento y control metabólico, así como la diabetes gestacional recurrente y el desarrollo de diabetes mellitus tipo 2.

Palabras clave: diabetes gestacional, diabetes mellitus, insulina, embarazo, glucosa.

Abreviaturas: DG, diabetes gestacional; DM, diabetes mellitus; DMG, diabetes mellitus gestacional: IN, insulina.

\section{GESTATIONAL DIABETES}

\section{Abstract}

Gestational diabetes affects 2-15 percent of pregnancies. Risk factors include obesity, impaired glucose tolerance, family history revealing diabetes mellitus and antecedents of a previous pregnancy resulting in fetal death. Diagnosis is based on two abnormal values in the oral glucose tolerance test. Medical management is based on a hypocaloric diet and exercise. Pharmacologic therapy with insulin has been traditional. The use of fast onset insulin analogues was approved in 1996. Follow-up and metabolic control, as well as relapses of gestational diabetes and the development of type 2 diabetes mellitus are herein analyzed.

Key words: gestational diabetes, DM, diabetes mellitus; GDM, gestational diabetes mellitus: IN, insulin.

Fecha recibido:marzo 16 de 2011 - Fecha aceptado: agosto 8 de 2011

* Ginecóloga y obstetra, Hospital de San josé. Instructora Asociada, Facultad de Medicina, Fundación Universitaria de Ciencias de la Salud, Bogotá DC. Colombia.
** Residente IV de Ginecología y Obstetricia, Fundación Universitaria de Ciencias de la Salud. Bogotá DC. Colombia. 


\section{Introducción}

La diabetes gestacional se define como cualquier grado de intolerancia a la glucosa que se inicia durante el embarazo. No excluye la posibilidad de que esta intolerancia no se haya reconocido antes del embarazo. Entre $2 \%$ y $15 \%$ de todas las gestaciones se ven complicadas por esta patología, que resulta en más de 200.000 casos por año. ${ }^{1,2}$ A menudo se considera como DM tipo 2 desenmascarada por el embarazo. Las dos entidades comparten factores de riesgo, algunos de los cuales van en aumento en la prevalencia, como obesidad y edad materna avanzada. Estos factores, combinados con un muestreo universal, han dado lugar a una mayor incidencia de DM. ${ }^{1,3}$

\section{Fisiopatología}

El embarazo normal es un estado diabetógeno debido al incremento progresivo de los niveles de glucosa posprandial y la mayor respuesta a la IN en los estadios tardíos de la gestación. ${ }^{4}$ Se caracteriza por un aumento adaptativo en la función de las células pancreáticas para compensar la disminución y sensibilidad a la IN y los elevados requerimientos, lo cual ocurre entre $40 \%$ y $70 \%$, en especial en el tercer trimestre. ${ }^{4,5}$

Los cambios en la acción de la IN condicionan la adaptación del metabolismo materno para favorecer la disposición de los nutrientes que requiere el feto en su desarrollo; en esta forma se establece un estado de resistencia a la IN que genera una mayor producción con el fin de compensarla, siempre que exista suficiente reserva funcional pancreática. En la DG no existe un déficit absoluto de la secreción de IN, pero sí alteraciones cualitativas de la misma. ${ }^{3,4,6}$

Los cambios en la resistencia a la IN durante el embarazo se relacionan con la concentración creciente de las hormonas placentarias, como lactógeno placentario, hormona de crecimiento, progesterona, cortisol y prolactina, los cuales se reducen después del parto. ${ }^{3}$ Hay un marcado aumento en las concentraciones de IN en ayunas y menor supresión de la producción hepática de glucosa durante la infusión de IN, lo que indica un descenso de la sensibilidad hepática en mujeres con DG. ${ }^{4}$

\section{Riesgo para la madre y el feto}

El propósito del diagnóstico temprano de DG es tratar a las pacientes lo antes posible en el embarazo, evitando así las posibles complicaciones tanto maternas y fetales como neonatales, asociadas con niveles elevados de glucosa en la sangre durante el embarazo, tales como macrosomía fetal, elevado porcentaje de parto por cesárea, alteraciones metabólicas neonatales y mayores tasas de ingreso a UCI, mortalidad perinatal, trastornos hipertensivos en la madre o sufrir DM tipo 2 después del parto. ${ }^{3,5,7}$

La macrosomía es uno de los problemas con mayor relación con DG y muchos autores utilizan como valor de corte para el peso al nacer que se halle por encima del percentil 90 ó que el recién nacido pese más de 4.000 gramos. La incidencia de macrosomía en mujeres con DMG es del $16 \%$ al $29 \%$, comparada con $10 \%$ en las mujeres sin la enfermedad..$^{2,6,8,9}$

Por otra parte, la DG parece no ser el único factor responsable del desarrollo de fetos macrosómicos. De todos los bebés con un peso mayor de 4.000 gramos sólo el 5\% de las madres tienen diagnóstico de la enfermedad, el resto son secundarios a la edad materna, el peso previo de la madre y durante el embarazo, así como la paridad..$^{2,6,10}$

La macrosomía es un resultado intermedio que por sí sola no es perjudicial para la madre o el recién nacido; sin embargo, puede ser causa del aumento de cesáreas, partos instrumentados, trauma del plexo braquial, fractura de clavícula o hipoglicemia neonatal. ${ }^{2,3,11}$ La tasa de cesáreas en DG relacionada con macrosomía es de cerca de $30 \%$, en comparación con $20 \%$ en aquellas sin esta patología. ${ }^{2,6}$

La distocia de hombros y el trauma al nacimiento, en especial la fractura de clavícula y las lesiones del plexo braquial, son desenlaces en los cuales está bien establecido que se relacionan en forma directa con el peso al nacer y el parto vaginal instrumentado; existen revisiones en las cuales la fractura de clavícula se ha encontrado entre $1,3 \%$ y $6 \%$ de los recién nacidos de madres con DG. ${ }^{3,6,7.12}$ La lesión del plexo braquial, 
aunque es una complicación que se presenta con menor frecuencia, no se debe dejar de lado dado pues es grave y puede dejar secuelas permanentes en $5 \%$ a $22 \%$ de los casos. ${ }^{13}$

Se ha observado una mayor proporción de hipoglicemia neonatal en hijos de madres diabéticas $(23 \%$ contra $0 \%$ en controles), así como también hiperbilirrubinemia, hipocalcemia y policitemia, con un mayor porcentaje de ingreso a UCI neonatal. Todo indica que si estas alteraciones son tratadas en forma adecuada, no tendrán efectos sobre el bebé y las complicaciones a largo plazo debidas a la hipoglicemia neonatal parecen limitarse a pocos casos, en los cuales es tan severa que puede producir convulsiones o hipoglicemia recurrente ${ }^{6,14,15}$

La mortalidad perinatal es la complicación más grave asociada con DG, aunque los reportes en la literatura cuentan con muy pocos casos, por lo cual se requieren estudios aleatorios con mayor población para poder llegar a una conclusión al respecto. ${ }^{3,6}$ La DG se ha asociado con aumento de los trastornos hipertensivos, con una incidencia tan elevada como del $20 \%$. Parece que a pesar de un adecuado manejo de la diabetes no se consigue una reducción significativa de los trastornos hipertensivos en el embarazo. , $7,8,14^{-}$

\section{Diagnóstico}

ElcuartotallerinternacionaldeDGen 1998recomendóuna estrategia de cribado, basada en la detección de factores de riesgo para diabetes desde el primer control prenatal, para clasificar a las pacientes como de bajo, intermedio y alto riesgo. ${ }^{16}$ Estas últimas deben ser tamizadas para DG tan pronto como sea posible; si las pruebas iniciales son negativas para diabetes, deben repetirse entre las semanas 24 y 28 de gestación. ${ }^{6,17-19}$

Los factores de riesgo más importantes son recién nacido macrosómico, obesidad, intolerancia a la glucosa, historia familiar de DM tipo 2 ó DG, antecedente de óbito fetal y en especial historia de diabetes en embarazos anteriores. ${ }^{20,21}$ El diagnóstico se basa en la curva de tolerancia a la glucosa con dos valores anormales, la cual por lo general se realiza después de una glicemia posprandial anormal de una hora, previa carga con 75 g por vía oral. ${ }^{3,722-25}$.

Las directrices emitidas por el cuarto taller internacional de DMG, la Asociación Americana de Diabetes y el Colegio Americano de Obstetras y Ginecólogos en el año 2001, recomiendan el uso de los criterios de Carpenter-Coustan para el de diagnóstico con carga de 100 g TTOG (curva de tolerancia a la glucosa, Tabla 1). ${ }^{16,19,26,27}$ Sin embargo, es importante

\begin{tabular}{|c|c|c|c|c|}
\hline & Glucometría & I h & $2 \mathrm{~h}$ & $3 \mathrm{~h}$ \\
\hline $\begin{array}{l}100 \mathrm{mg} \\
\text { TTOG* } \\
\text { C\&C }{ }^{26}\end{array}$ & $95 \mathrm{mg} / \mathrm{dl}$ & $180 \mathrm{mg} / \mathrm{dl}$ & $155 \mathrm{mg} / \mathrm{dl}$ & $140 \mathrm{mg} / \mathrm{dl}$ \\
\hline $\begin{array}{l}100 \mathrm{mg} \\
\text { TTOG } \\
\text { NDDG }{ }^{28}\end{array}$ & $105 \mathrm{mg} / \mathrm{dl}$ & $190 \mathrm{mg} / \mathrm{dl}$ & $165 \mathrm{mg} / \mathrm{dl}$ & $145 \mathrm{mg} / \mathrm{dl}$ \\
\hline $\begin{array}{l}75 \mathrm{mg} \\
\text { TTOG } \\
\text { WHO } \neq^{29}\end{array}$ & $126 \mathrm{mg} / \mathrm{dl}$ & & $140 \mathrm{mg} / \mathrm{dl}$ & \\
\hline $\begin{array}{l}75 \mathrm{mg} \\
\text { TTOG } \\
\text { ADA } \mathbb{1}^{4}\end{array}$ & $95 \mathrm{mg} / \mathrm{dl}$ & 180 mg/dl & 155 mg/dl & \\
\hline $\begin{array}{l}75 \mathrm{mg} \\
\text { TTOG } \\
\text { CDAS }\end{array}$ & $95 \mathrm{mg} / \mathrm{dl}$ & $190 \mathrm{mg} / \mathrm{dl}$ & $160 \mathrm{mg} / \mathrm{dl}$ & \\
\hline
\end{tabular}

* TTOG, curva de tolerancia a la glucosa; †NDDG, National Diabetes Date Group; $\ddagger$ Organización Mundial de la Salud; IIADA, Asociación Americana de Diabetes; §CDA, Asociación Canadiense de Diabetes. 
recordar que al igual que otras pruebas de detección, las utilizadas para DG pueden tener problemas de reproducibilidad..$^{1,3,16,19}$

\section{Manejo médico}

El tratamiento inicial para la DG sigue siendo la dieta y el ejercicio, recomendándose una hipocalórica de 1.900 a $2.400 \mathrm{kcal} /$ día con restricción de carbohidratos de $35 \%$ a $40 \%$ de las calorías (calculada con base en el peso corporal ideal antes del embarazo) y administración de complejos ricos en fibra. ${ }^{31}$ La dieta adecuada aplaza la terapia farmacológica. La mayoría de las mujeres pierden peso durante las primeras semanas de dieta, pero a continuación se reanuda una modesta ganancia. $^{21,31}$

Si el ejercicio no está contraindicado por otras complicaciones obstétricas, puede mejorar el control glicémico. Durante la actividad física, el músculo estriado consume glucosa entre 2 y $3 \mathrm{mg} / \mathrm{k}$ de peso por minuto de ejercicio; el nivel sérico previo al inicio del ejercicio debe ser de 90 a $140 \mathrm{mg} / \mathrm{dl}$, habrá consumido una comida una a tres horas antes de comenzar la sesión y esta no debe durar más de 45 minutos. ${ }^{6,21}$ Si la paciente se encuentra en manejo con IN, el bolo preprandial previo al ejercicio debe disminuirse en un $50 \%$ de la dosis acostumbrada. ${ }^{32}$ Lo recomendado consiste en caminatas, bicicleta estática, aeróbicos de bajo impacto y natación; cada una debe ser precedida por una sesión de calentamiento, seguido por una fase de resistencia durante la cual la frecuencia cardíaca no debe exceder 140 latidos por minuto. ${ }^{21,32}$

\section{Terapia farmacológica}

Se establece cuando la dieta y el ejercicio no han conseguido control adecuado de más de la mitad de los valores de las glucometrías. ${ }^{6,27,33} \mathrm{La}$ insulina ha sido el fármaco de elección debido a su seguridad durante el embarazo y el reducido paso transplacentario. La mayoría de las pacientes pueden tratarse en forma ambulatoria. ${ }^{33-35}$
Los análogos de insulina de acción rápida disponibles en el mercado, como la insulina lispro, se aprobaron por primera vez en 1996; son el resultado de la tecnología de recombinación del ADN y consisten en una modificación de la cadena beta de la insulina humana. Esta IN se disocia rápido en subunidades monoméricas cuando se inyecta en el tejido subcutáneo, presenta una acción rápida debido a una absorción más eficaz a través de la membrana capilar en comparación con la insulina regular y su uso está asociado con una reducción de 27 a $36 \mathrm{mg} / \mathrm{dl}$ de la glicemia posprandial, con una mejoría de la hemoglobina glicosilada. Además, se ha identificado que disminuyen en $24 \%$ los episodios de hipoglicemia en ayunas (por debajo de $55 \mathrm{mg} / \mathrm{dl}$ ) y presentan menores tasas de hipoglicemia posprandial menor de $120 \mathrm{mg} / \mathrm{dl}$ en suero una hora posprandial en comparación con la IN regular. ${ }^{34,36,37}$

La IN aspártica, aprobada para uso clínico en 1999, se crea mediante la sustitución de prolina en la posición B28, reduce la glicemia posprandial en cerca de 27 $\mathrm{mg} / \mathrm{dl}$, los de hemoglobina glicosilada en un $0,12 \%$ y disminuye los episodios graves de hipoglicemia en un $50 \%$ en comparación con la IN regular. ${ }^{34,38} \mathrm{Si}$ el manejo de la DG está dirigido a un adecuado control metabólico valorado por los niveles de glicemia posprandial, la IN lispro de acción rápida y la IN aspartato parecen ser tan seguras y efectivas como la IN humana regular. ${ }^{34,39}$

La dosis inicial de IN en embarazo se basa en el peso materno y se calcula determinando las necesidades totales diarias: $0,8 \mathrm{U} / \mathrm{k}$ de peso corporal en el primer trimestre; 1 , U/k en el segundo y $1,2 \mathrm{U} / \mathrm{k}$ en el tercero. ${ }^{34,35}$ Una vez determinada la dosis total diaria de IN se administran dos tercios antes del desayuno, divididos en dos tercios de NPH y uno de IN regular, y el tercio restante de la dosis diaria se divide en la mitad de IN regular antes de la cena y la otra mitad de IN NPH a la hora de acostarse. ${ }^{34,37}$ La IN de acción de muy corta duración se dosifica mejor con cada comida, a diferencia de la IN regular que es dos veces al día.

En el siglo XXI, los hipoglicemiantes orales se han incluido en el arsenal de modalidades de tratamiento para la DG, encontrando en diferentes estudios que 
las mujeres tratadas con gliburida en comparación con IN, tienen más probabilidades de alcanzar el objetivo de normalización de los valores de glicemia tanto en ayunas como posprandial; además, los recién nacidos tienen peso similar a los de madres sanas y son menos propensos a remitirse a la UCI neonatal. ${ }^{35,40,41}$

La gliburida parece no atravesar la placenta en estudios realizados in vitro; sin embargo, otros investigadores han encontrado niveles de este medicamento en cordón umbilical de $0,7+/-0.4 \mathrm{mg} / \mathrm{dl}$ lo que sugiere que sí hay paso. ${ }^{40}$ Las fallas del tratamiento con gliburida se pueden predecir pues son más probables en mujeres con diagnóstico temprano en el embarazo, mayores de 35 años de edad, multíparas y con altas cifras de glicemia en ayunas, lo que refleja una función reducida de las células beta y de la capacidad de respuesta de los secretagogos de IN.$^{6,27}$ Estos factores deben considerarse antes de iniciar el tratamiento con gliburida, sin olvidar que no hay probabilidades de alcanzar las metas si el nivel de glucosa en ayunas es mayor de $140 \mathrm{mg} / \mathrm{dl} .{ }^{6.39}$

Al comparar el control glucémico de la gliburida con la metformina como agentes únicos para el manejo de la DG, la tasa de fracasos para alcanzar las metas de glicemia posprandial es 2.1 veces mayor para la metformina. Por lo demás, los resultados maternos y neonatales son similares entre los dos grupos. Se ha evidenciado que el $46,3 \%$ de las pacientes que reciben metformina como fármaco inicial requieren IN adicional para lograr euglucemia. ${ }^{42-44} \mathrm{El}$ menor peso al nacer ha sido sugerido como un beneficio adicional de la terapia con metformina debido a su acción como un sensibilizador de IN y su capacidad para atravesar la placenta. ${ }^{42,43}$

\section{Seguimiento y control metabólico}

El quinto taller internacional sobre DG recomienda que las concentraciones de glucosa en sangre en ayunas se encuentren entre 90 y $99 \mathrm{mg} / \mathrm{dl}$, la sérica posprandial a la hora menor de $140 \mathrm{mg} / \mathrm{dl}$ y a las dos horas entre 120 y $127 \mathrm{mg} / \mathrm{dl} .^{6}$
Los niveles de hemoglobina glicosilada basal y de intervalo durante el tratamiento son útiles, sobre todo en quienes tienen hiperglicemia en ayunas. La mayoría de las mujeres con terapia dietaria se deben monitorizar con glucometrías cuatro veces al día (glicemia en ayunas y posprandial, tres veces al día). Aquellas con tratamiento farmacológico requieren control cuatro a seis veces al día, incluyendo los valores antes de las comidas. ${ }^{6}$ El monitoreo semanal en el consultorio y los procedimientos diarios parecen tener resultados comparables en cuanto a morbimortalidad perinatal.5,6 Las mujeres con DG tratadas con dieta que realizan autocontrol glucométrico, son menos propensas a tener un recién nacido macrosómico. ${ }^{21}$ Se ha visto que hay una reducción significativa del peso materno cuando las pacientes notan niveles altos de glucosa en sangre en la automonitorización; se sienten más motivadas a participar en su propio cuidado, además de mostrarse más receptivas con respecto a las intervenciones en la dieta. ${ }^{5,45,46}$

Sin embargo, no se han demostrado diferencias importantes en el peso de los recién nacidos en relación con el monitoreo intensivo y el convencional. ${ }^{11,34,45,47}$ Aunque el control glucémico juega un papel determinante en el tamaño del feto, el exceso de peso materno también influye en forma significativa; así, es evidente que una reducción en la ganancia de peso materno es beneficiosa, incluso en mujeres obesas. El mecanismo de esta interacción es incierto. ${ }^{21,45}$

\section{Diabetes gestacional recurrente}

Dado que la mayoría de los factores de riesgo para DG persisten o empeoran en los siguientes embarazos, no es de extrañar que esta patología tenga una alta tasa de recurrencia, del 35,6\% al 70\%. ${ }^{1,6}$ Las mujeres con embarazos consecutivos complicados con diabetes tienen fetos con más peso para la edad gestacional y es más común que requieran IN durante el embarazo (38\% comparadas con $19 \%$ en las que no desarrollan la enfermedad). ${ }^{1,48,49}$

La recurrencia de DG es más frecuente en pacientes con más de un embarazo, índice de masa corporal mayor de $30 \mathrm{k} / \mathrm{m}^{2}$ al inicio de la gestación, diagnóstico 
de DG en el embarazo previo antes de las 24 semanas, así como el requerimiento de IN. Un aumento de peso de más de $10 \mathrm{k}$ y un periodo integenésico menor de 24 meses también están asociados, aunque en menor proporción. ${ }^{1,48}$

\section{Desarrollo de diabetes mellitus tipo 2}

Las mujeres con antecedente de DG presentan mayor posibilidad de desarrollar DM tipo 2 en un futuro, tal vez por tener en común factores de riesgo asociados; la incidencia varía en los diferentes estudios entre $2 \%$ y $70 \%$, de acuerdo con el tiempo de seguimiento (6-8 semanas a 28 años). ${ }^{50,51}$ La detección temprana de características que indiquen alto riesgo de padecer diabetes, como el sobrepeso y la historia familiar, permite un control adecuado para prevenir el desarrollo de esta enfermedad. ${ }^{33,50,52}$

Las mujeres que cursan con DG en un alto porcentaje presentan resistencia crónica a la IN, la cual se acentúa y se hace más evidente durante el embarazo, comparada con quienes no desarrollan la enfermedad durante el mismo. ${ }^{1,50,51}$ Se ha encontrado que la primera fase de secreción de IN durante el embarazo es un factor predictivo importante para la posterior aparición de DM tipo $2 .{ }^{33}$ Dentro de los factores de riesgo que se asocian con DM están la multiparidad, glicemia en ayunas elevada en la gestación, sobrepeso previo al embarazo, índice de masa corporal, la exagerada ganancia de peso durante la gestación y la presencia de hiperglicemia durante cuatro a 16 semanas después del parto. ${ }^{33}$

La Asociación Americana de Diabetes recomienda la evaluación del estado glucémico para todas las mujeres con DMG seis semanas después del parto. ${ }^{33}$

Según una estimación reciente, alrededor de $10 \%$ a $31 \%$ de DM en las multíparas se puede atribuir a la presencia de DG previa. ${ }^{50}$

No hay ningún estándar universal aceptado para la evaluación del metabolismo de la glucosa en las mujeres que han tenido DMG. Sin embargo, es importante tener una estrategia de tres fases de seguimiento (después del parto, los primeros meses posparto y a largo plazo). Aunque los niveles de glucosa circulante por lo regular regresan a la normalidad después del parto, esto puede no suceder en aquellas con DM tipo 2 preexistente que se identificó por primera vez durante el embarazo. ${ }^{48,50,51}$

Una evaluación seis a doce semanas después del parto proporciona información que es de gran valor para la salud y planificación de la familia, así como el alto riesgo de progresión a DM tipo 2 en los siguientes cinco a diez años; está justificado tener una estrategia para continuar con el seguimiento. ${ }^{48}$

La Asociación Americana de Diabetes recomienda el uso de una prueba de glucosa sérica en ayunas para la evaluación y clasificación durante el puerperio, mientras que en la quinta conferencia taller internacional sobre DMG puso en evidencia que la glicemia en ayunas como herramienta de diagnóstico posparto no identificó una proporción importante de las que cursaban con intolerancia a la glucosa o DM; solo el $44 \%$ con DG y curva de tolerancia a la glucosa alterada tenían una glicemia fuera de los rangos normales; por lo tanto, las recomendaciones hacen hincapié en el uso de TTGO para la evaluación del metabolismo de la glucosa después del parto en DG. ${ }^{48,50}$

\section{Manejo antenatal e intraparto}

Una vez que se diagnostica DG, la embarazada debe ser vista a intervalos de una a dos semanas, con más frecuencia si se presentan complicaciones. En general, mujeres en control dietario en las cuales no se haya documentado un peso fetal por encima del percentil 90 , pueden esperar hasta las 40 semanas y el riesgo de muerte fetal no es mayor que el de la población general. Las que han requerido manejo farmacológico, se ha documentado feto grande o con restricción del crecimiento, o tiene otras complicaciones del embarazo, requieren seguimiento estrecho, pero el momento para la inducción de parto es aun controvertido dado que no existen recomendaciones específicas. ${ }^{6}$ La conducta actual es ofrecer a las mujeres con DG en quienes se estima un peso fetal mayor o igual a 4.000 gramos, 
parto por cesárea electiva para prevenir la distocia de hombros. ${ }^{6}$

\section{Prevención de la DM después del embarazo}

Los beneficios potenciales con la prevención o retraso en la aparición de la DM tipo 2 en pacientes de alto riesgo está bien documentada. En el Programa de Prevención de la Diabetes, tanto la modificación en el estilo de vida como el uso de metformina redujeron la progresión de intolerancia a la glucosa a DM, tanto en mujeres como en hombres, así como en el subgrupo de aquellas con antecedente de DG. Las reducciones fueron del $58 \%$ asociadas con cambios en el estilo de vida, donde el objetivo estaba orientado a una pérdida del $7 \%$ del peso corporal inicial y actividad física de al menos 150 minutos por semana y $31 \%$ para metformina, $850 \mathrm{mg}$ dos veces al día. ${ }^{48,50}$

\section{Referencias}

1. Bottalico JN. Recurrent gestational diabetes: risk factors, diagnosis, management, and implications. Semin Perinatol. 2007 Jun;31 (3):176-84.

2. Landon MB, Spong CY, Thom E, Carpenter MW, Ramin SM, Casey B, et al. A multicenter, randomized trial of treatment for mild gestational diabetes. $\mathrm{N}$ Engl J Med. 2009 Oct 1;361(14):1339-48.

3. Metzger BE, Buchanan TA, Coustan DR, de LA, Dunger DB, Hadden DR, et al. Summary and recommendations of the Fifth International Workshop-Conference on Gestational Diabetes Mellitus. Diabetes Care. 2007 Jul;30 Suppl 2:S251S260.

4. Gabbe N\&S. Ginecología y obstetricia. 1 ed. 2007.

5. Kjos SL. After pregnancy complicated by diabetes: postpartum care and education. Obstet Gynecol Clin North Am. 2007 Jun;34(2):335-49, x.

6. Pridjian G, Benjamin TD. Update on gestational diabetes. Obstet Gynecol Clin North Am. 2010 Jun;37(2):255-67.

7. Coustan DR. Lowe LP, Metzger BE, Dyer AR. The Hyperglycemia and Adverse Pregnancy Outcome (HAPO) study: paving the way for new diagnostic criteria for gestational diabetes mellitus. Am J Obstet Gynecol. 2010 Jun;202(6):654-6.

8. Naylor CD, Sermer M, Chen E, Sykora K. Cesarean delivery in relation to birth weight and gestational glucose tolerance: pathophysiology or practice style? Toronto Trihospital Gestational Diabetes Investigators. JAMA. 1996 Apr 17;275(15):1165-70.

9. George C. Lu MDa DRMMDaSCB. The impact of lower threshold values for the detection of gestational diabetes mellitus. Obstetrics \& Gynecology. 2000 Apr;44.

10. Ayach W, Calderón IM, Rudge MV, Costa RA. [Comparison between two gestational diabetes screening tests and the perinatal outcome]. Rev Bras Ginecol Obstet. 2010 May;32(5):222-8.

11. Witkop CT, Neale D, Wilson LM, Bass EB, Nicholson WK. Active compared with expectant delivery management in women with gestational diabetes: a systematic review. Obstet Gynecol. 2009 Jan;113(1):206-17.

12. Ecker JL, Greenberg JA, Norwitz ER, Nadel AS, Repke JT. Birth weight as a predictor of brachial plexus injury. Obstet Gynecol. 1997 May;89(5 Pt 1):643-7.
13. Jevitt CM. Shoulder dystocia: etiology, common risk factors, and management. J Midwifery Womens Health. 2005 Nov;50(6):485-97.

14. Jensen DM, Sorensen B, Feilberg-Jorgensen N, Westergaard JG, Beck-Nielsen H. Maternal and perinatal outcomes in 143 Danish women with gestational diabetes mellitus and 143 controls with a similar risk profile. Diabet Med. 2000 Apr;17(4):281-6.

15. González-Quintero VH, Istwan NB, Rhea DJ, Rodríguez LI, Cotter A, Carter J, et al. The impact of glycemic control on neonatal outcome in singleton pregnancies complicated by gestational diabetes. Diabetes Care. 2007 Mar;30(3):467-70.

16. Metzger BE, Coustan DR. Summary and recommendations of the Fourth International Workshop-Conference on Gestational Diabetes Mellitus. The Organizing Committee. Diabetes Care. 1998 Aug; 21 Suppl 2:B161-B 167.

17. Smirnakis KV, Plati A, Wolf M, Thadhani R, Ecker JL. Predicting gestational diabetes: choosing the optimal early serum marker. Am J Obstet Gynecol. 2007 Apr; 196(4):410-6.

18. Paglia MJ, Coustan DR. Gestational diabetes: evolving diagnostic criteria. Curr Opin Obstet Gynecol. 2010 Dec 20.

19. Lois Jovanovic. Global guideline on pregnancy and diabetes, IDF Guidelines. IDF guidelines 2009Available from: URL: www.idf.org

20. Serlin DC, Lash RW. Diagnosis and management of gestational diabetes mellitus. Am Fam Physician. 2009 Jul 1;80(1):57-62.

21. Kari D Pilolla, Melinda M Manore. Gestational Diabetes mellitus. The other dia betes on the rise. Acm Healt \& Fitness Journal. 2008;12(5):8-13.

22. Alberti KG, Zimmet PZ. Definition, diagnosis and classification of diabetes mellitus and its complications. Part 1: diagnosis and classification of diabetes mellitus provisional report of a WHO consultation. Diabet Med. 1998 Jul; 15(7):539-53.

23. ACOG Practice Bulletin. Clinical management guidelines for obstetrician-gynecologists. Number 30, September 2001 (replaces Technical Bulletin Number 200, December 1994). Gestational diabetes. Obstet Gynecol. 2001 Sep;98(3):525-38.

24. Lee AJ, Hiscock RJ, Wein P, Walker SP, Permezel M. Gestational diabetes mellitus: clinical predictors and long-term risk of developing type 2 diabetes: a retrospective cohort study using survival analysis. Diabetes Care. 2007 Apr;30(4):878-83.

25. Esakoff TF, Cheng YW, Sparks TN, Caughey AB. The association between birthweight $4000 \mathrm{~g}$ or greater and perinatal outcomes in patients with and without gestational diabetes mellitus. Am J Obstet Gynecol. 2009 Jun;200(6):672-4.

26. Cheng YW, Block-Kurbisch I, Caughey AB. Carpenter-Coustan criteria compared with the national diabetes data group thresholds for gestational diabetes mellitus. Obstet Gynecol. 2009 Aug;114(2 Pt 1):326-32.

27. Hollander MH, Paarlberg KM, Huisjes AJ. Gestational diabetes: a review of the current literature and guidelines. Obstet Gynecol Surv 2007 Feb;62(2):125-36.

28. Classification and diagnosis of diabetes mellitus and other categories of glucose intolerance. National Diabetes Data Group. Diabetes. 1979 Dec;28(12):1039-57.

29. Silva JK, Kaholokula JK, Ratner R, Mau M. Ethnic differences in perinatal outcome of gestational diabetes mellitus. Diabetes Care. 2006 Sep;29(9):2058-63.

30. Meltzer S, Leiter L, Daneman D, Gerstein HC, Lau D, Ludwig S, et al. 1998 clinical practice guidelines for the management of diabetes in Canada. Canadian Diabetes Association. CMAJ. 1998;159 Suppl 8:S1-29.

31. Nutrition Recommendations and Interventions for Diabetes: a position statement of the American Diabetes Association. Diabetes Care. 2007 Jan;30 Suppl 1:S48-S65.

32. Unger J. Management of diabetes in pregnancy, childhood, and adolescence. Prim Care. 2007 Dec;34(4):809-43, vii.

33. Krishnaveni GV, Hill JC, Veena SR, Geetha S, Jayakumar MN, Karat CL, et al. Gestational diabetes and the incidence of diabetes in the 5 years following the index pregnancy in South Indian women. Diabetes Res Clin Pract. 2007 Dec;78(3):398-404.

34. Singh C, Jovanovic $\mathrm{L}$. Insulin analogues in the treatment of diabetes in pregnancy. Obstet Gynecol Clin North Am. 2007 Jun;34(2):275-91, ix. 
35. Nicholson W, Bolen S, Witkop CT, Neale D, Wilson L, Bass E. Benefits and risks of oral diabetes agents compared with insulin in women with gestational diabetes: a systematic review. Obstet Gynecol. 2009 Jan;113(1):193-205.

36. Jovanovic L, Ilic S, Pettitt DJ, Hugo K, Gutiérrez M, Bowsher RR, et al. Metabolic and immunologic effects of insulin lispro in gestational diabetes. Diabetes Care. 1999 Sep;22(9):1422-7.

37. Bhattacharyya A, Brown S, Hughes S, Vice PA. Insulin lispro and regular insulin in pregnancy. QJM. 2001 May;94(5):255-60

38. Pettitt DJ. MPOMJWKMPaLJM. Comparison of an Insulin Analog, Insulin Aspart, and Regular Human Insulin With No Insulin in Gestational Diabetes Mellitus. Diabetes Care. 2003 Jan;26(1):183-6.

39. Vidaeff AC, Yeomans ER, Ramin SM. Gestational diabetes: a field of controversy. Obstet Gynecol Surv. 2003 Nov;58(11):759-69.

40. Langer O, Conway DL, Berkus MD, Xenakis EM, Gonzales O. A comparison of glyburide and insulin in women with gestational diabetes mellitus. N Engl J Med. 2000 Oct 19;343(16):1134-8

41. Langer O. Oral anti-hyperglycemic agents for the management of gestational diabetes mellitus. Obstet Gynecol Clin North Am. 2007 Jun;34(2):255-74, ix.

42. Moore LE, Clokey D, Rappaport VJ, Curet LB. Metformin compared with glyburide in gestational diabetes: a randomized controlled trial. Obstet Gynecol. 2010 Jan;1 15(1):55-9.

43. Rowan JA, Gao W, Hague WM, McIntyre HD. Glycemia and its relationship to outcomes in the metformin in gestational diabetes trial. Diabetes Care. 2010 Jan;33(1):9-16.

44. Balani J, Hyer SL, Rodin DA, Shehata H. Pregnancy outcomes in women with gestational diabetes treated with metformin or insulin: a case-control study. Diabet Med. 2009 Aug;26(8):798-802.
45. Hawkins JS, Casey BM, Lo JY, Moss K, McIntire DD, Leveno KJ. Weekly compared with daily blood glucose monitoring in women with diet-treated gestational diabetes. Obstet Gynecol. 2009 Jun;113(6):1307-12.

46. Heuberger RPR. Increasing Empathy, Knowledge, and Skills Regarding Gestational Diabetes. Topics in Clinical Nutrition. 2010 Sep;25(3):250-5.

47. Athukorala C, Crowther CA, Willson K. Women with gestational diabetes mellitus in the ACHOIS trial: risk factors for shoulder dystocia. Aust N Z J Obstet Gynecol. 2007 Feb;47(1):37-41

48. Metzger BE. Long-term outcomes in mothers diagnosed with gestational diabetes mellitus and their offspring. Clin Obstet Gynecol. 2007 Dec;50(4):972-9.

49. Paramsothy P, Lin YS, Kernic MA, Foster-Schubert KE. Interpregnancy weight gain and cesarean delivery risk in women with a history of gestational diabetes. Obstet Gynecol. 2009 Apr;113(4):817-23.

50. Schaefer-Graf UM, Buchanan TA, Xiang AH, Peters RK, Kjos SL. Clinical predictors for a high risk for the development of diabetes mellitus in the early puerperium in women with recent gestational diabetes mellitus. Am J Obstet Gynecol. 2002 Apr;186(4):751-6

51. Smimakis KV, Chasan-Taber L, Wolf M, Markenson G, Ecker JL, Thadhani R. Postpartum diabetes screening in women with a history of gestational diabetes. Obstet Gynecol. 2005 Dec;106(6):1297-303.

52. Simmons D, McElduff A, McIntyre HD, Elrishi M. Gestational diabetes mellitus: NICE for the U.S.? A comparison of the American Diabetes Association and the American College of Obstetricians and Gynecologists guidelines with the U.K. National Institute for Health and Clinical Excellence guidelines. Diabetes Care. 2010 Jan;33(1):34-7. 